\title{
Assessment of residual chlorine in soil microbial community using metagenomics
}

\author{
Yitian $\mathrm{Yu}^{1,}$, Qi Zhang ${ }^{1,}$, Zhenyan Zhang ${ }^{1}$, Nuohan $\mathrm{Xu}^{1}$, Yan $\mathrm{Li}^{1}$, Mingkang $\mathrm{Jin}^{2,3}$, Guoqiang Feng ${ }^{4}$, \\ Haifeng Qian', Tao Lu' ${ }^{1, *}$
}

1 College of Environment, Zhejiang University of Technology, Hangzhou 310014, China

2 Key Laboratory of Urban Environment and Health, Institute of Urban Environment, Chinese Academy of Sciences, Xiamen 361021, China 3 University of Chinese Academy of Sciences, Beijing 100049, China

4 School of Economics, Lanzhou University, Lanzhou 730000, China

\section{H I G H L I G H T S}

- Continuous chlorine treatment have no obvious effect on soil microbial community structure and composition.

- Residual chlorine slightly affected soil microbial functions.

- Daily use of chlorine-containing disinfectants slightly threatened the soil ecosystem.
GRAPHICAL ABSTRACT

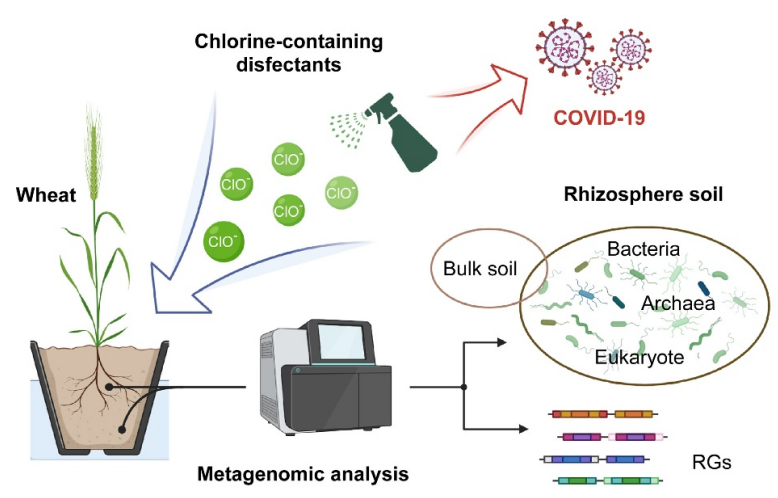

Article history:

Received September 25, 2021

Revised November 17, 2021

Accepted November 25, 2021

Keywords:

Soil microbes

Chlorine-containing disinfectants

Plant microbiome

Metagenome

Sodium hypochlorite

\section{A B S T R A C T}

Chlorine-containing disinfectants have been widely used around the world for the prevention and control of the COVID-19 pandemic. However, at present, little is known about the impact of residual chlorine on the soil micro-ecological environment. Herein, we treated an experimental soil-plantmicrobiome microcosm system by continuous irrigation with a low concentration of chlorinecontaining water, and then analyzed the influence on the soil microbial community using metagenomics. After 14-d continuous chlorine treatment, there were no significant lasting effect on soil microbial community diversity and composition either in the rhizosphere or in bulk soil. Although metabolic functions of the rhizosphere microbial community were affected slightly by continuous chlorine treatment, it recovered to the original status. The abundance of several resistance genes changed by $7 \mathrm{~d}$ and recovered by $14 \mathrm{~d}$. According to our results, the chlorine residue resulting from daily disinfection may present a slight long-term effect on plant growth (shoot length and fresh weight) and soil micro-ecology. In general, our study assisted with environmental risk assessments relating to the application of chlorine-containing disinfectants and minimization of risks to the environment during disease control, such as COVID-19.

(c) Higher Education Press 2022

\footnotetext{
* Corresponding author

E-mail address: lutao@zjut.edu.cn (T. Lu)

'These authors contributed equally to this work.
} 


\section{Introduction}

The COVID-19 epidemic is sweeping around the world and is even considered a long-term war between viral disease and humans (Duarte et al., 2020; WHO, 2020a). To establish a healthy barrier and reduce the transmission of COVID-19, the use of disinfectants is essential. Among them, chlorinecontaining disinfectants, mainly sodium hypochlorite are commonly used products in emergency health events owing to high disinfection efficiency and low cost. It has been recommended that surface decontamination infected by COVID-19 infection should be controlled (Wang et al., 2020; Subpiramaniyam, 2021). The WHO has recommended that to effectively control COVID-19 or other diseases, the residual chlorine in the drinking water distribution system must be kept $>0.5 \mathrm{mg} \mathrm{L}^{-1}$ (WHO, 2020b). Meanwhile, numerous chlorinecontaining disinfectants were used for continuous public disinfection, such as in areas with severe epidemics. According to some sources, the usage of chlorine-containing disinfectants reached 1963.58 tons in the first two months during the COVID-19 epidemic in Wuhan, China (China Huanqiu Web, 2020). As a result, the continuous application and overuse of chlorine-containing disinfectants inevitably led to serious ecological pollution in the environment (Choi et al., 2020; Chu et al., 2021; Zhang et al., 2021), especially in soil ecosystems directly sprayed with chlorine-containing disinfectants (Mincarelli et al., 2016).

Soil microorganisms, especially those colonizing the plant rhizosphere, play an important role in growth yield and disease-resistance of plant (Lu et al., 2018; Qu et al., 2020; Feng et al., 2021), but most soil ecotoxicology experiments have usually focused on big-size model organisms for study but have ignored the soil microbial ecology. The indiscriminate spraying of a large amounts of chlorine-containing disinfectants can directly destroy the community balance and functional diversity of soil microbiome, which may further perturb the health of plants and result in invasion by pathogenic bacteria (Mendes et al., 2013; Qian et al., 2021). Previous studies on the effects of chlorination in irrigation water on plants and epiphytic microorganisms provided a research basis for the risk assessment of chlorine-containing disinfectants residual on the soil environment (Lonigro et al., 2017; Truchado et al., 2019). For example, Hao et al. (2018) found that long-term agricultural irrigation with chlorinated water had a slight impact on soil enzyme activity but indicated that it was safe for field crops with precipitation leaching. Similarly, the $\mathrm{ClO}_{2}$ treatment of irrigation water did not affect the diversity of water, soil, or crop bacterial communities. However, significant differences were observed in the relative abundance of specific bacteria belong to Enterobacteriaceae and Pseudomonas (Truchado et al., 2018). However, until now, the impact of chlorine disinfectants on soil microbial community has been mainly concentrated at the level of community composition, and the change of functions often cannot be determined accurately and comprehensively. Fortunately, the development of metagenomics has enhanced the understanding of the microbial community composition and its specific ecological functions.

Meanwhile, the development of biotechnology has enabled the assessment of the ecotoxicity of pollutants the level of the more complex microbial community rather than that of a single organism, allowing for comprehensive ecotoxicity assessment resulting from human interference. Herein, we selected the model plant wheat (Triticum aestivum L.) to construct the soil-plant-microbiome microcosm system and simulated continuous exposure to chlorine-containing disinfectants. We aimed to comprehensively assess the ecological risk of disinfectant in soil during the COVID-19 pandemic using metagenome.

\section{Materials and methods}

\subsection{Establishment of soil-plant-microbiome microcosms}

Wheat (Triticum aestivum L., Yannong 19) seeds were soaked in $0.6 \%$ nitric acid solution for $10 \mathrm{~min}$ to release them from dormancy and make them germinate effectively. The seeds were then disinfected with $75 \%$ ethanol and $2.5 \%$ calcium hypochlorite for $1 \mathrm{~min}$ and $15 \mathrm{~min}$, respectively, and rinsed with sterile water 6-7 times. The surface-sterilized seeds were left in a dark environment at $30^{\circ} \mathrm{C}$ for $7 \mathrm{~d}$ to germinate, transferred to a plastic pot containing about $230 \mathrm{~g}$ of soil (including $30 \mathrm{~g}$ of sterilized water), and a soil suspension made of vegetable garden soil (from 30¹7'45.11" N, $\left.120^{\circ} 09^{\prime} 50.07^{\prime \prime} \mathrm{E}\right)$ was then added as the source of rhizosphere microorganisms (vegetable garden soil:sterile water = $30: 200)$. The obtained soil-plant-microbiome microcosms were placed at $25^{\circ} \mathrm{C}$, and $80 \%$ relative humidity, with $12 \mathrm{~h}$ light/12 $\mathrm{h}$ dark cycle at a light intensity of $300 \mu \mathrm{mol}$ photons $\mathrm{m}^{-2} \mathrm{~s}^{-1}$ for a week.

\subsection{Chlorine treatment of soil-plant-microbiome microcosms}

The sodium hypochlorite disinfectant (Bluemoon, Guangzhou, China) used in this study was purchased from a local supermarket during the COVID-19 epidemic, and its active chlorine content was $8000 \mathrm{mg} \mathrm{L}^{-1}$ detected by multiparameter water quality detector (LH-M900, Hangzhou LuHeng Biological Technology Co.). The stock solution was diluted with deionized water to the different final concentrations of active chlorine $\left(0.05\right.$ and $\left.1 \mathrm{mg} \mathrm{L}^{-1}\right)$ and added to the soil-plant-microbiome microcosms in three replicates daily for $14 \mathrm{~d}$. $20 \mathrm{~mL}$ of different concentrations mentioned above of chlorine solution were added into each treated group per day. The same amount of deionized water was added in the control group.

\subsection{Soil sample collection}

The bulk and rhizosphere soil of wheat were collected $7 \mathrm{~d}$ and $14 \mathrm{~d}$ after seedling transplantation (the vegetable garden soil 
used to make the soil suspension having been sampled on day 0). The soil sample was collected as described by Bulgarelli et al. (2012). In detail, the upper soil was gently collected with a spoon as a bulk soil to explore the direct effects of chlorine treatment on the composition and functions of the soil microbial community. Then, the rhizosphere was gently shaken to remove loose and large pieces of soil from the roots, and the soil samples attached to the root surface were collected as rhizosphere soil for further study. All samples were stored at $-80^{\circ} \mathrm{C}$ immediately after collection.

\subsection{Measurement of soil enzyme activity}

Parts of bulk and rhizosphere soil sample were dried at $37^{\circ} \mathrm{C}$, then ground and screened (60-mesh). Next, acid phosphatase (ACP), urease, and sucrase activities in the microcosm soil were determined using enzyme activity kits (Nanjing Jiancheng Bioengineering Institute, Nanjing) according to the manufacturer's instructions. The concentration of enzymes in samples was measured using a visible spectrophotometer (Shanghai INESA Analytical Instrument Co., Ltd., Shanghai); the measurement wavelengths of ACP, urease, and sucrase were 405, 578, and $540 \mathrm{~nm}$, respectively. The hydrolysis of $\mathrm{p}$ nitrophenyl phosphate (pNPP) per hour per gram of soil sample producing $1 \mathrm{nmol}$ p-nitrophenol (PNP) $\left(37^{\circ} \mathrm{C}\right)$ was defined as a unit of ACP activity. The production of $1 \mu \mathrm{g} \mathrm{NH} \mathrm{N}_{3}-\mathrm{N}$ per gram of soil sample per day was set as a unit of urease activity, whereas the production of $1 \mathrm{mg}$ reducing sugar per gram of soil sample per day was considered as a unit of sucrase activity.

\subsection{Microcosm metagenome sample preparation and sequencing}

The metagenomic analysis of bulk and rhizosphere soil microbiomes at 0, 7 and $14 \mathrm{~d}$ after treatment were performed in three replicates. The total genomic DNA of these samples was isolated using a DNA extraction kit (ALFA-SEQ Advanced Water DNA Kit, Guangdong Magigene Biotechnology Co., Ltd., Guangzhou, China) according to the manufacturer's instructions. The concentration and purity of the extracted soil DNA were determined using Qubit 2.0 (Thermo Fisher Scientific, Waltham, USA) and NanoDrop One (Thermo Fisher Scientific, Waltham, USA). A DNA library preparation kit was used to generate the sequence libraries (NEB Next Ultra DNA Library Prep Kit, New England Biolabs, MA, USA) following the manufacturer's instructions, and the library quality was verified using Qubit 3.0 fluorometer (Life Technologies, Grand Island, NY). Next, the DNA sequencing was performed on an Illumina Novaseq platform (Illumina, CA, USA).

\subsection{Microcosm metagenome assembly and analysis}

After filtering the detected low-quality bases in each metagenome data set (Trimmomatic v0.36), the obtained clean data were used for subsequent analysis (Bolger et al., 2014). Next, the de novo assembly of clean data was performed on MEGAHIT v1.0.6 (Li et al., 2015), and each assembled scaftig (>500 bp) was predicted to open reading frames (ORFs) using Prodigal (https://github.com/hyattpd/Prodigal). Meanwhile, the redundant ORFs were removed using CD-HIT v4.7 (Fu et al., 2012) to obtain the unique initial gene (unigene) clusters (95\% identity with $90 \%$ coverage), and the longest sequences were selected as representative of each cluster. In addition, clean data of each sample were mapped to unigenes using BBMap to obtain the number of reads to which unigenes mapped in each sample. Based on the number of mapped reads and the length of unigene, the abundance of unigene $i$ in sample $\mathrm{S}$ was calculated as:

$$
\mathrm{RPKM}_{i}=\frac{x_{i}}{L_{i}(\mathrm{~kb}) \times x_{\text {total }}(\text { millions })}
$$

where $\mathrm{RPKM}_{i}$ (Reads Per Kilobase Million) was the abundance of unigene $i$ in the sample $S, x_{i}$ was the number of mapped reads for unigene $i$ in sample $S, x_{\text {total }}$ was the total number of all mapped reads in the sample $S$ and $L_{i}$ was the length of unigene $i$.

The nonredundant (NR) database of NCBI and the Kyoto Encyclopedia of Genes and Genomes (KEGG) (http://www. kegg.jp/kegg/) databases were employed to perform a BLAST search for taxonomic and functional annotations, respectively, using the DIAMOND software (https://github.com/bbuchfink/ diamond/). The LCA algorithm in MEGAN (Huson et al., 2007) was utilized to calculate the abundance and each taxonomic classification (kingdom, phylum, class, order, family, genus, species) based on the alignment of NR unigenes with an evalue $\leqslant 1 \mathrm{e}-10$. With regards to the functional analysis, the functional hierarchy level and relative abundance were determined using the best BLAST hit (lowest e-value).

\subsection{Statistics and visualization}

The mean values and standard errors in each group were determined using Microsoft Excel 365 (Microsoft Corporation, Redmond, WA, USA). The alpha diversities (Shannon index and richness) of rhizosphere and bulk soil microbial community were calculated at the genus level using the vegan package in R (Dixon, 2003) and visualized by GraphPad Prism 8.0.2. Principal coordinate analysis (PCoA) was performed based on the Bray-Curtis dissimilarity at the genus level using the vegan and ggplot2 packages in $\mathrm{R}$. The heatmaps showing the difference in soil microbiome and associated function abundance (KEGG database), were drawn by TBtools (Toolbox Biologists v0.655). The significance of differences between treatment and control was calculated with a two-tailed T.TEST in Microsoft Excel 365, where a $p$-value $<0.05$ was defined as significant. The relationships between 103 significantly changed genera and 30 significantly changed functions were analyzed and visualized as co-occurrence network. The correlations between genera and functions were analyzed using pairwise 
Spearman's rank correlations $(r)$ in the psych package of $\mathrm{R}$. We picked out strong $(|r|>0.8)$ and significant $(p<0.05)$ correlations and visualized the data using Cytoscape (version 3.8.0).

\section{Results}

\subsection{Effect of chlorine on activity of microcosm soil enzymes}

Before the formal experiment, wheat plants were exposed to five different concentrations $\left(0,1,3,10\right.$, and $\left.30 \mathrm{mg} \mathrm{L}^{-1}\right)$ of chlorine treatment. The shoot length and fresh weight of plants were determined, and the results indicated that even high concentration of chlorine treatment had little effect on wheat growth (Fig. S1). Besides, since soil enzymatic activity can roughly reflect the ecological functions of soil microbial communities (Cui et al., 2021), two concentrations of chlorine treatment ( 1 and $10 \mathrm{mg} \mathrm{L}^{-1}$ ) were selected for the determination of enzymatic activity. The activities of solid-acid phosphatase (ACP), urease, and sucrase in rhizosphere and bulk soil after 7-d and 14-d treatment are shown in Fig. 1. Neither concentration of chlorine ( 1 and $10 \mathrm{mg} \mathrm{L}^{-1}$ ) had a significant effect on the three enzymatic activities in bulk soil during the 14-d treatment period. However, in rhizosphere soil, the urease activity significantly increased (two-tailed T.TEST, $p<0.05$ ) under $10 \mathrm{mg} \mathrm{L}^{-1}$ chlorine treatment by $7 \mathrm{~d}$, and ACP activity also significantly elevated (two-tailed T.TEST, $p<0.05$ ) under $1 \mathrm{mg} \mathrm{L}^{-1}$ chlorine treatment by $14 \mathrm{~d}$. In rhizosphere soil, high-concentration chlorine treatment (10 $\mathrm{mg} \mathrm{L}^{-1}$ ) presented a short-term influence on $\mathrm{N}$ utilization, while low-concentration chlorine treatment $\left(1 \mathrm{mg} \mathrm{L}^{-1}\right)$ had a long-term influence on $\mathrm{P}$ utilization; the bulk soil microbiome was barely affected by chlorine treatment. Additionally, the physiological indicators (shoot height and fresh weight) of wheat presented a slight response to continuous chlorine treatment (Fig. S2; two-tailed T.TEST, $p>0.05$ ).

\subsection{Effect of chlorine on diversity and structure of soil microbial community}

To more directly reflect the disturbance of a soil microbial community caused by chlorine treatment, the metagenome was used to characterize the soil microbial community diversity and composition. Based on the results of enzymatic activity and actual soil environmental chlorine concentrations, the 0.05 and $1 \mathrm{mg} \mathrm{L}^{-1}$ chlorine treatment were selected for exposure and subsequent metagenomic analysis. PCoA with Bray-Curtis dissimilarity of the genera abundance indicated that both concentrations of chlorine treatment have a slight impact on the rhizosphere microbial community composition by $14 \mathrm{~d}$ (Adonis analysis, $R^{2}=0.3880, p=0.034$ ) (Fig. 2). Consistently with the result of enzymatic activity, no significant differences were observed in bulk microbial community composition (Adonis analysis, $R^{2}=0.2469, p>0.05$ ) (Fig. S3A, B). Similarly, the Shannon and richness indices, calculated based on genus level data, showed that there was no significant difference between rhizosphere and bulk microbial diversity with or without chlorine treatment (Fig. S3C, D). Consequently, chlorine had little effect on the microbial community diversity and composition.

\subsection{Effect of chlorine on microbial community composition}

To further investigate the effects of chlorine on microbial community composition, the composition of microbial phyla and genera was analyzed. The results of microbial composition at phylum level showed that Proteobacteria, Euryarchaeota, and Ascomycota were the dominated bacteria, archaea, and eukaryotes, respectively (Fig. 3A). Among the bacterial phyla, Proteobacteria, Acidobacteria, Actinobacteria, Chloroflexi, and Bacteroidetes were the top five most abundant groups. There was no significant difference in the bacterial abundance after chlorine treatment.

According to composition of microbial genera, we found that Sphingomonas (Phylum: Proteobacteria), Nocardioides (Phylum: Actinobacteria), and Chryseolinea (Phylum: Bacteroidetes) were abundant both in rhizosphere and bulk soil during the 14-d chlorine treatment period (Fig. 3B). In comparison with the control, the rhizosphere soil had 361 and 289 genera changed in abundance in the $0.05 \mathrm{mg} \mathrm{L}^{-1}$ of chlorine treatment at $7 \mathrm{~d}$ and $14 \mathrm{~d}$, respectively; 360 and 210 genera changed in the $1 \mathrm{mg} \mathrm{L}^{-1}$ of chlorine treatment at $7 \mathrm{~d}$ and $14 \mathrm{~d}$, respectively; a total of 123 and 305 genera in bulk soil changed in the $0.05 \mathrm{mg} \mathrm{L}^{-1}$ of chlorine treatment at $7 \mathrm{~d}$ and $14 \mathrm{~d}$, respectively; a total of 288 and 221 genera changed in the $1 \mathrm{mg} \mathrm{L}^{-1}$ of chlorine treatment at $7 \mathrm{~d}$ and $14 \mathrm{~d}$, respectively (Fig. $3 \mathrm{C}$ ). Overall, in both concentrations of chlorine treatment $\left(0.05\right.$ and $\left.1 \mathrm{mg} \mathrm{L}^{-1}\right), 70$ genera showed significant changes (two-tailed T.TEST, $p<0.05$ ) in abundance at $7 \mathrm{~d}$, and 39 genera at $14 \mathrm{~d}$ (Fig. S4). For example, Novosphingobium, Porphyrobacter, Croceicoccus, and Metallibacterium were significantly decreased (two-tailed T.TEST, $p<0.05)$ under both concentrations of chlorine treatment at both $7 \mathrm{~d}$ and $14 \mathrm{~d}$ (Fig. 3D). Thus, the composition analysis of microbial species showed that chlorine impacted the abundance of certain microbe species slightly.

\subsection{Effect of chlorine on microbial functions}

Based on the above results, the rhizosphere microbiota exhibited more significant changes under chlorine treatment than bulk soil, therefore, the KEGG function analysis helped us to further explore the impact of chlorine on rhizosphere microbiota functions. The functions of the rhizosphere microbial community were significantly (two-tailed T.TEST, $p<0.05$ ) changed by chlorine treatment at $7 \mathrm{~d}$ and $14 \mathrm{~d}$, as shown in Figs. 4 and S5. Overall, the number of significantly different pathways at $7 \mathrm{~d}$ was slightly higher than that at $14 \mathrm{~d}$ (Fig. S5). Interestingly, 8 and 24 functions under $0.05 \mathrm{mg} \mathrm{L}^{-1}$ chlorine treatment significantly differed from the control at $7 \mathrm{~d}$ and $14 \mathrm{~d}$, respectively, while 23 and 6 functions under 

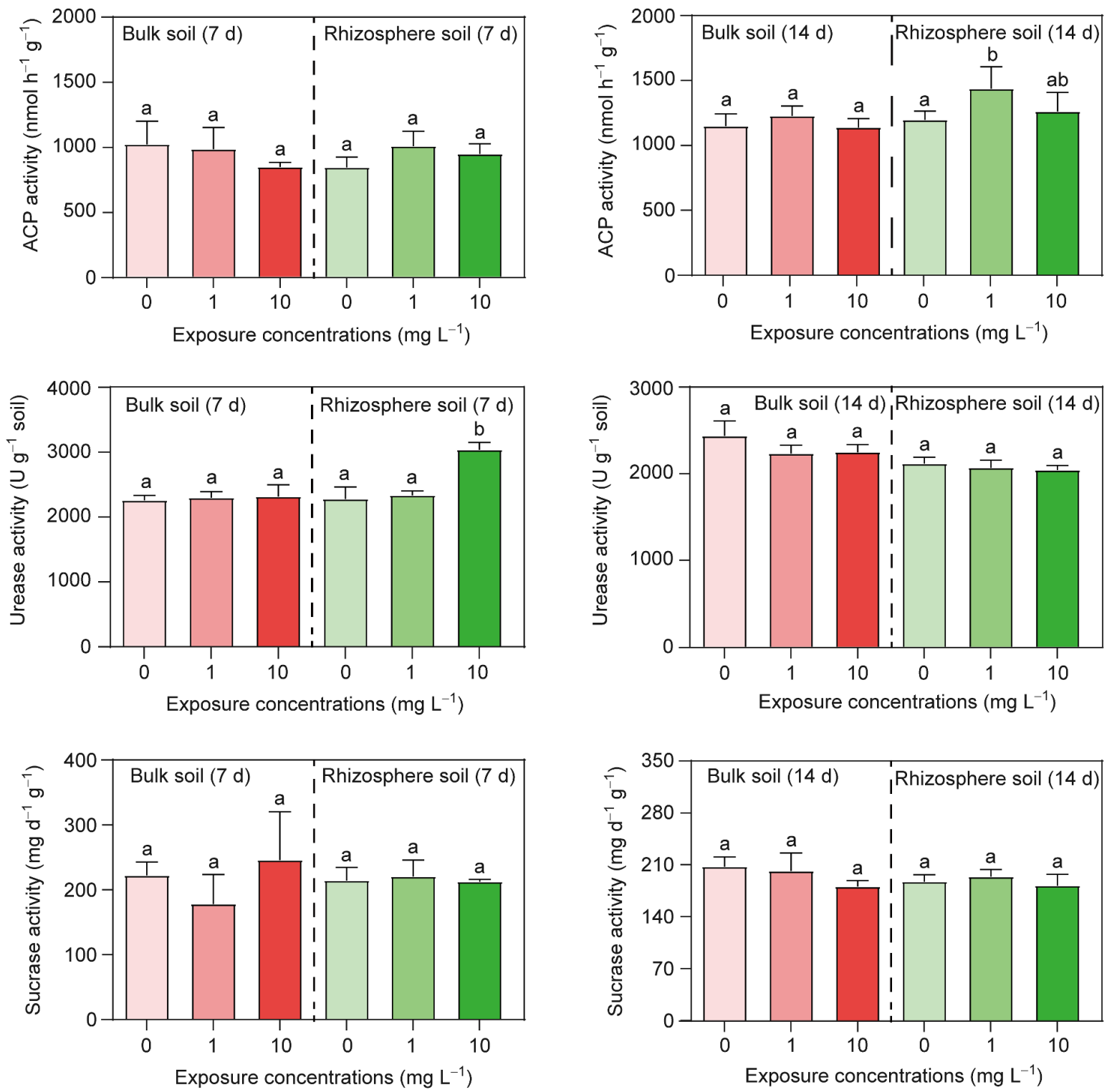

Fig. 1 The activity of solid-acid phosphatase (ACP), urease, and sucrase of rhizosphere and bulk soil after 7-d and 14-d treatment. Every column represents the mean \pm SE with four replicates. Different letters indicate a significant difference (two-tailed T.TEST, $p<0.05$ ).

$1 \mathrm{mg} \mathrm{L}^{-1}$ chlorine treatment significantly differed from the control at $7 \mathrm{~d}$ and $14 \mathrm{~d}$, respectively (Fig. 4).

Plenty of functions associated with metabolism enriched sharply after 7 -d treatment with $1 \mathrm{mg} \mathrm{L}^{-1}$ chlorine and decreased notably after $14-\mathrm{d}$ treatment with $0.05 \mathrm{mg} \mathrm{L}^{-1}$ chlorine (Fig. 4). Meanwhile, functions associated with cellular processes, oocyte meiosis (ko04114) and focal adhesion (ko04510), as well as tight junction (ko04530), had decreased after $7 \mathrm{~d}$ of $1 \mathrm{mg} \mathrm{L}^{-1}$ chlorine treatment. Furthermore, basal transcription factors (ko03022), the function associated with genetic information processing, was strongly affected by chlorine. The trend of three functions, ko02010, ko04371 and ko04150, associated with environmental information processing performed similarly to metabolism, cellular processes, and genetic information processing mentioned above, respectively. Therefore, we speculated that a high concentration of chlorine might represent a short-term influence while a low concentration of chlorine might constitute a long-term influence, and both concentrations of chlorine disturbed mainly metabolism.

3.5 Correlation analysis of significant different bacteria and functions

The correlation between significantly changed KEGG functions and bacteria abundance was calculated based on Spearman's rank correlation coefficients (Fig. 5 and Fig. S6). A total of 8 phyla and 10 functions were strongly correlated (Spearman's $|r|>0.8, p<0.05$ ), of which were 14 

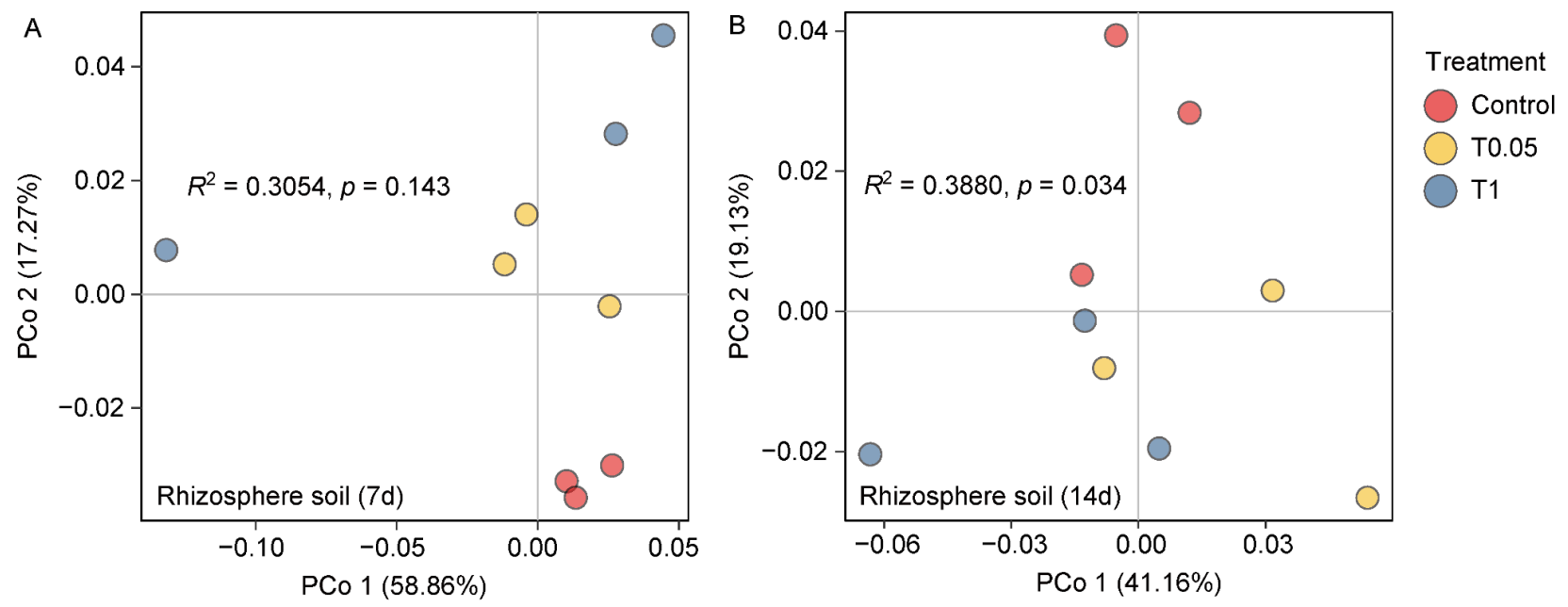

Fig. 2 Changes of rhizosphere microbial community diversity and structure in chlorine treatment. The initial microbial communities of each group are the same (day 0). PCoA analysis of microbe genera communities using Bray-Curtis distance of different chlorine concentration treatments after $7 \mathrm{~d}$ or $14 \mathrm{~d}$ in rhizosphere soil. T0.05 and T1 represent low and high concentrations of chlorine treatment, respectively.

positive and 59 were negative correlations. For example, Mobiluncus (Phylum: Actinobacteria) and Soehngenia (Phylum: Firmicutes) were positively correlated (Spearman's $|r|$ $>0.8, p<0.05$ ) with cell growth and death (ko04114); Acidovorax (Phylum: Proteobacteria) was positively correlated (Spearman's $|r|>0.8, p<0.05$ ) with $\mathrm{ABC}$ transporters (ko02010). Moreover, Haloactinobacterium (Phylum: Actinobacteria) was notable positively correlated (Spearman's $|r|$ $>0.8, p<0.05$ ) with all of: metabolism (ko00230: purine metabolism, ko00310: Iysine degradation, ko00340: histidine metabolism, ko00400: phenylalanine, tyrosine and tryptophan biosynthesis, and ko00750: vitamin B6 metabolism), genetic information processing (ko03022: basal transcription factors), and environmental information processing (ko02010: ABC transporters). In a similar manner, Brevibacterium (Phylum: Actinobacteria) was strongly positively correlated (Spearman's $|r|>0.8, p<0.05$ ) with metabolism (ko00340: histidine metabolism and ko00400: phenylalanine, tyrosine and tryptophan biosynthesis), environmental information processing (ko02010: ABC transporters), and cellular processing (ko04114: oocyte meiosis).

\subsection{Effect of chlorine on abundance of resistance genes (RGs)}

RGs were annotated using raw sequence data based on the BacMet database, including antibacterial biocides and metal resistance genes. In rhizosphere soil, among the top 50 high abundant genes, a total of 10 and 9 RGs were significantly affected by chlorine at $7 \mathrm{~d}$ and $14 \mathrm{~d}$, respectively (Fig. 6). Principal coordinates analysis (PCoA) of rhizosphere soil at $7 \mathrm{~d}$ showed that the abundance of RGs in the control were clustered and separated from those in the $1 \mathrm{mg} \mathrm{L}^{-1}$ chlorine treatment along the PCo1 axis (explaining $93.49 \%$ of the variation; Fig. S7). Besides, the result of Adonis test indicated that the abundance of RGs in control was significantly different from that in chlorine treatment $(p<0.05$; Fig. S7).
Meanwhile, in bulk soil, among the top 50 most abundant genes, there were only 8 and 2 genes significantly different at $7 \mathrm{~d}$ and $14 \mathrm{~d}$ respectively (Fig. 6). However, PCoA based on Bray-Curtis distances and Adonis test showed that there was no significant alteration in the abundance of RGs of bulk soil after chlorine treatment ( $p>0.05$; Fig. S7).

\section{Discussion}

Due to the wide application of sprayed chlorine-containing disinfectants within short time periods, chlorine can directly contact the soil and soil microorganisms, especially those of the rhizosphere. Rhizosphere microbiota plays a crucial role in the growth and disease resistance of plants (Mendes et al., 2013; Qu et al., 2020). Therefore, it is essential to assess the potential risks presented by disinfectants to the soil ecological environment. In this study, we established soil-plant-microbiome microcosms and demonstrated the effects of continuous chlorine treatment on the microbial communities of wheat rhizosphere and bulk soil using metagenomics analysis.

Soil acid phosphatase (ACP) could decompose organic phosphorus compounds to produce phosphate that was used by plants and microorganisms, and thus plays a key role in the soil phosphorus cycle (Olander and Vitousek, 2000; Qian et al., 2007). The level of urease, which could degrade urea (an important nitrogen fertilizer) to $\mathrm{CO}_{2}$ and $\mathrm{NH}_{3}$, reflected the soil nitrogen content, and has been shown to be highly sensitive to soil pollution (Hu et al., 2013; Hao et al., 2018). In this study, the effect of chlorine on soil enzyme activity was related to the type of soil enzyme, the concentration of chlorine treatment, and the treatment time. Our results showed that highconcentration (10 $\mathrm{mg} \mathrm{L}^{-1}$ ) chlorine treatment caused urease activity to increase first and then decline, while low-concentration $\left(1 \mathrm{mg} \mathrm{L}^{-1}\right)$ chlorine treatment made ACP higher than in the control group at $14 \mathrm{~d}$, even though these two effects were 

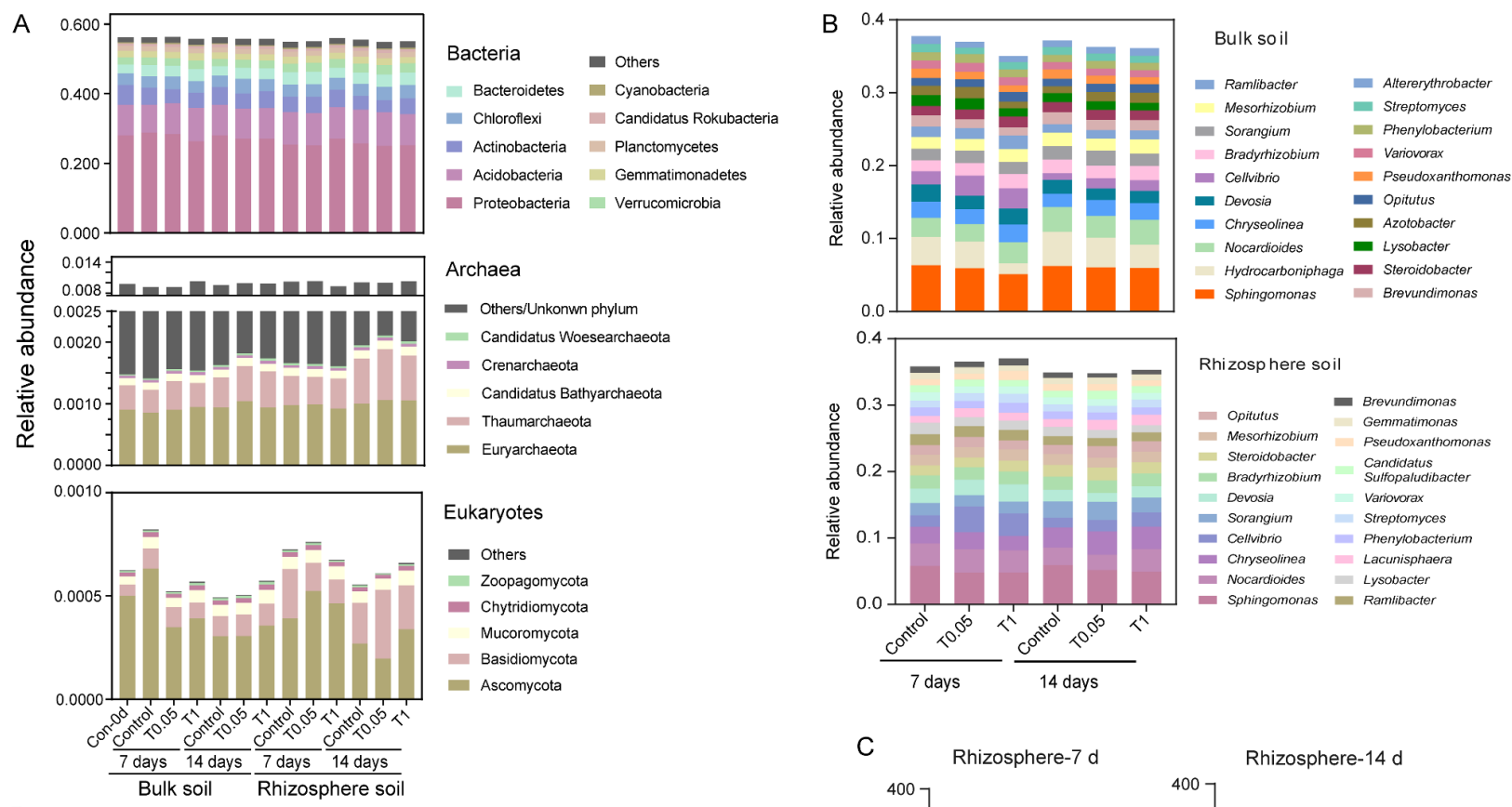

D
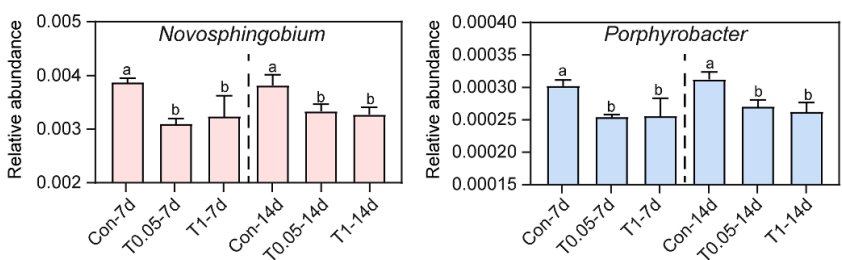

C
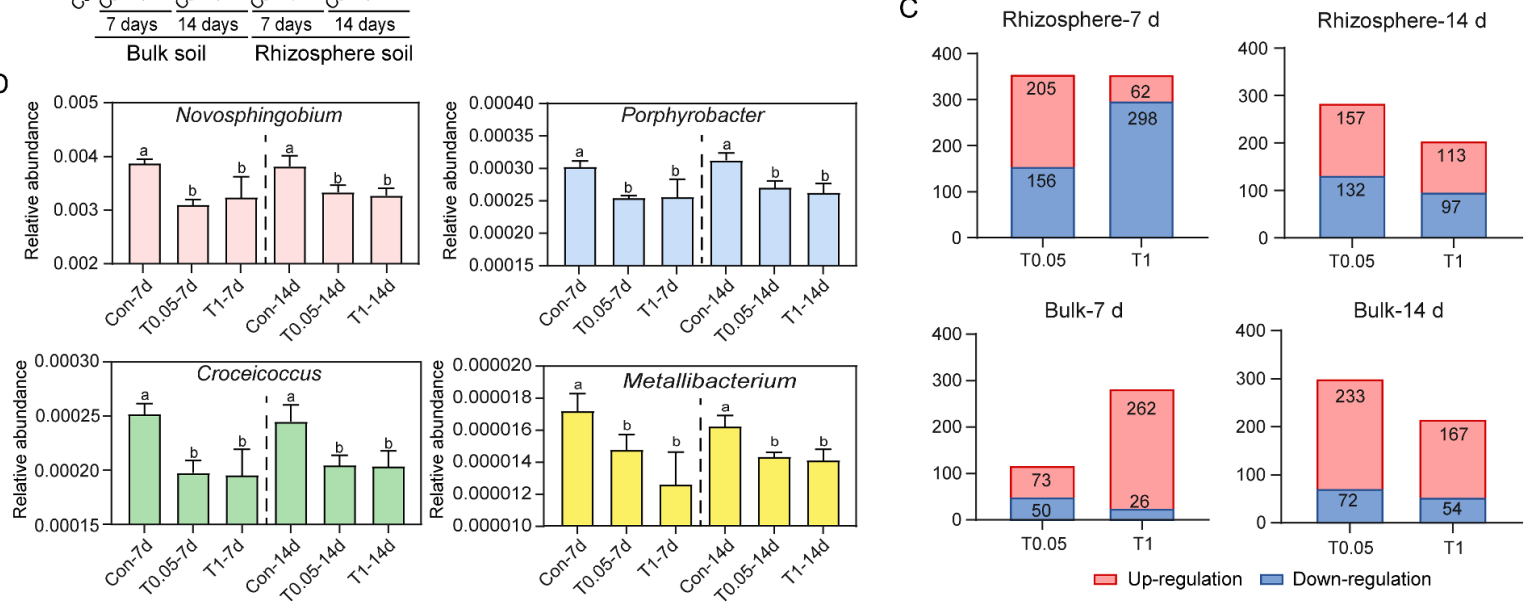

Fig. 3 Effect of chlorine treatment on microbial composition at the phylum and genus level. (A) Composition of bacteria (top 10 in abundance), archaea (top 5 in abundance), and eukaryotes (top 5 in abundance) at phylum level. (B) Composition of soil microbiome at genus level (top 20 in abundance). (C) The number of enhanced and decreased genera which were significantly affected by chlorine (twotailed T.TEST, $p<0.05$ ). (D) Four genera significantly decreased in abundance (two-tailed T.TEST, $p<0.05$ ) as a result of chlorine treatment. Different letters indicate a significant difference (two-tailed T.TEST, $p<0.05$ ).

only slight. Studies have also shown that soil properties, such as in saline soil, fluvo-aquic soil, and red soil, affected the response of enzyme activity to pollutants (Hu et al., 2013). However, the change of soil enzyme activity cannot accurately reflect the alteration of soil microbiome in response to chlorine treatment.

Metagenomic analysis helped us better understand the impact of chlorine treatment on the soil microbial community. According to the results of pre-experiments, we selected two representative concentrations $\left(0.05\right.$ and $\left.1 \mathrm{mg} \mathrm{L}^{-1}\right)$ of irrigation water with chlorine to treat wheat, based on the actual amount of residual chlorine in the soil and the concentration of disinfectant in contact with the soil, which were within reasonable residual chlorine range (Truchado et al., 2018; Subpiramaniyam, 2021). In this study, after continuous chlorine treatment for $14 \mathrm{~d}$, the composition of the rhizosphere microbial community significantly differed from control group, while the bulk soil microbial community did not, which was consistent with the results of soil enzyme activity mentioned above. For example, the Novosphingobium (Phylum: Proteobacteria) became abundantly enriched in chlorine-treated rhizosphere and could utilize abscisic acid (ABA) as a carbon and energy source (Oleńska et al., 2020). Novosphingobium were considered as nitrogen-fixing plant-promoting endophytic bacteria (Zhang et al., 2016) that could effectively fix nitrogen in the soil (Rangjaroen et al., 2017) and promoted the growth of rice (Krishnan et al., 2017). Some rhizosphere bacteria with low abundance after chlorine treatment, such as Porphyrobacter (Phylum: Proteobacteria), Croceicoccus (Phylum: Proteobacteria), and Metallibacterium (Eosinophilic 


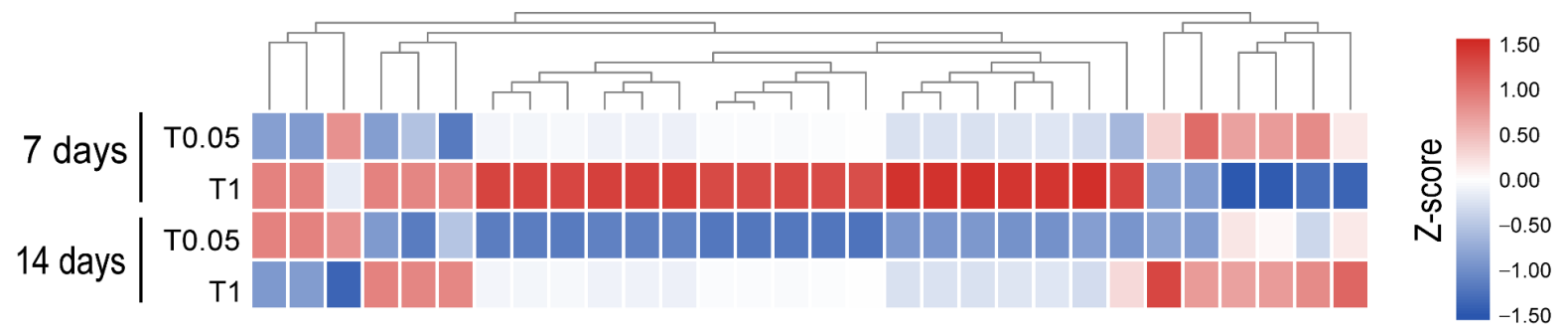

\section{Metabolism}

Global and overview maps

\begin{tabular}{ll|l|l|l|l}
\hline 0.00 & 0.04 & -0.03 & 0.00 & $k 001100$
\end{tabular}

\begin{tabular}{ll|l|l|l|l}
\hline 10.00 & 0.04 & -0.03 & 0.00 & $k 001110$
\end{tabular}

\begin{tabular}{c|c|c|c|c|c}
\hline-0.00 & 0.05 & -0.04 & 0.00 & $k 001212$
\end{tabular}

\begin{tabular}{c|c|c|c|c|c|}
\hline-0.00 & 0.05 & -0.04 & 0.00 & $k 001212$ \\
\hline 0.00 & 0.04 & -0.02 & 0.00 & $k 001230$
\end{tabular}

Energy metabolism

$\begin{array}{lllll}50.00 & -0.10 & 0.00 & -0.22 k 000196\end{array}$

Lipid metabolism

$\begin{array}{cccccc}\ulcorner & 0.00 & 0.06 & -0.04 & 0.00 & k 000071\end{array}$

Nucleotide metabolism

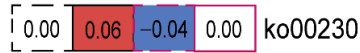

Amino acid metabolism

\begin{tabular}{lll|l|l|l}
$\Gamma 0.00$ & 0.04 & -0.03 & 0.00 & $k 000260$
\end{tabular}

\begin{tabular}{lll|l|l|l}
\hline 0.00 & 0.06 & -0.04 & 0.00 & $k 000310$
\end{tabular}

\begin{tabular}{ll|l|l|l|l}
\hline & 0.00 & 0.04 & -0.04 & 0.00 & $k 000330$
\end{tabular}

$\begin{array}{llllll}1-0.00 & 0.04 & -0.04 & 0.00 & k 000330\end{array}$

\begin{tabular}{lllllll}
\hline 0.00 & 0.06 & -0.02 & 0.00 & $k 000340$
\end{tabular}

\begin{tabular}{lllllll}
\hline & 0.00 & 0.06 & -0.02 & 0.00 & $k 000400$
\end{tabular}
Metabolism of other amino acids

\begin{tabular}{ll|l|l|l|l}
50.00 & 0.05 & -0.04 & 0.00 & $k 000410$
\end{tabular}

\begin{tabular}{llllll}
\hline 0.00 & 0.04 & -0.02 & 0.00 & $k 000460$
\end{tabular}

\begin{tabular}{llllll}
\hline-0.03 & 0.00 & -0.04 & 0.00 & $k 000471$
\end{tabular}

$\begin{array}{lllll}-0.08 & 0.00 & -0.05 & 0.00 & k 000472\end{array}$

Metabolism of cofactors and vitamins

\begin{tabular}{ll|l|l|l|l}
\hline 0.00 & 0.06 & -0.02 & 0.00 & $k 000750$ \\
\hline 0.00 & 0.05 & -0.04 & 0.00 & $k 000780$ \\
\hline & &
\end{tabular}

Metabolism of terpenoids and polyketides

$$
\left[\begin{array}{ll|l|l|l}
{[0.00} & 0.07 & -0.06 & 0.00 & k 000903
\end{array}\right.
$$

Biosynthesis of other secondary metabolites

$$
\begin{array}{ccccc}
-0.04 & 0.00 & -0.04 & 0.00 & k 000333
\end{array}
$$

Xenobiotics biodegradation and metabolism

\begin{tabular}{c|c|c|c|c}
{$[-0.06$} & 0.00 & 0.00 & -0.06 & $k 000364$ \\
-0.05 & 0.00 & 0.00 & -0.06 & $k 000623$ \\
-0.04 & 0.05 & -0.05 & 0.00 & $k 000930$ \\
\hline & & 0.00 & $k$
\end{tabular}

Environmental information processing

Membrane transport

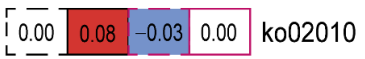

Signal transduction

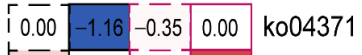

\begin{tabular}{lllllll}
\hline & 0.19 & 0.00 & 0.00 & 0.36 & $k 004150$
\end{tabular}

Genetic information processing

Transcription

\begin{tabular}{lll|l|l|l}
\hline 0.16 & 0.00 & 0.00 & 0.13 & k003022
\end{tabular}

Cellular processes

Cell growth and death

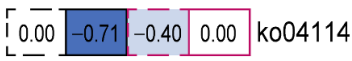

Cellular community - eukaryotes

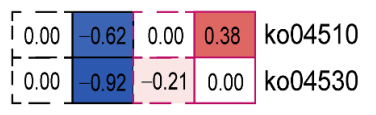

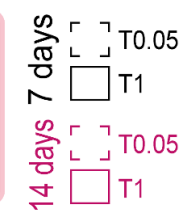

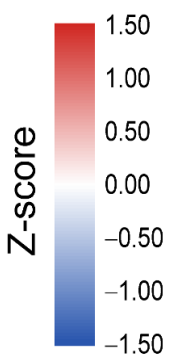

Fig. 4 Heatmaps show the significantly changed $(p<0.05)$ KEGG pathways of rhizosphere microbiota under different concentrations of

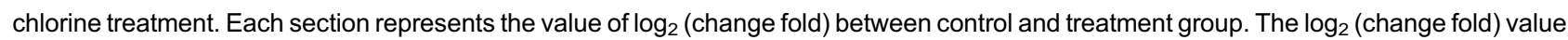
of pathways without significant difference was adjusted to 0 . And every $\log _{2}$ (change fold) was transformed to a Z-score.

iron metabolizing bacteria, Phylum: Proteobacteria), were also plant probiotic (Yurkov et al., 1994; Ziegler et al., 2013; Huang et al., 2015). Similarly, the abundance of Novosphingobium decreased significantly after $7 \mathrm{~d}$ and $14 \mathrm{~d}$ of continuous chlorine treatment. This finding demonstrated that continuous chlorine treatment perturbed the rhizosphere microbial community, especially the bacteria promoting plant growth. In contrast, compared with the control group, the abundance of rhizosphere bacteria Gilvimarinus and Luteimicrobium with much lower initial abundance was increased after continuous chlorine treatment. Previous study found that Gilvimarinus could produce certain hydrolases (such as polysaccharide hydrolase and agarase) to degrade macromolecular substances (such as cellulose and agar) (Shousei and Katsuhiko, 2018; Lee et al., 2018), and Luteimicrobium (Phylum: Actinobacteria) could colonize the termite intestine and produce protein substrate hydrolase (Murphy et al., 2016; Miwa et al., 2019). Thus, we speculated that plant roots might recruit and accumulate beneficial microbes to resist the stress caused by chlorine (Qu et al., 2020).
However, although the abundance of some microbes changed under chlorine stress, the microbial community diversity and composition did not change significantly after $14 \mathrm{~d}$ of continuous chlorine treatment. Therefore, we speculated that because the abundance of bacteria was far more than that of archaea and eukaryotes, and the abundance of soil dominant bacteria was affected little by chlorine treatment, so the overall effect of chlorine treatment on microbial community composition was not significant (Fig. 3B). Meanwhile, archaea and eukaryotes were not significantly affected by chlorine treatment (Fig. S8).

The above results indicated that the bulk microbial community was barely affected by chorine treatment, thus only the alteration of rhizosphere microbial community was considered in further analysis. Results related to the rhizosphere microbiome showed that the number of functions changed significantly under chlorine treatment at $7 \mathrm{~d}$, and slightly higher at $14 \mathrm{~d}$, of which reasons were that the chlorine treatment stimulated the rhizosphere microbial community in a short period of time, and the rhizosphere microbial community 


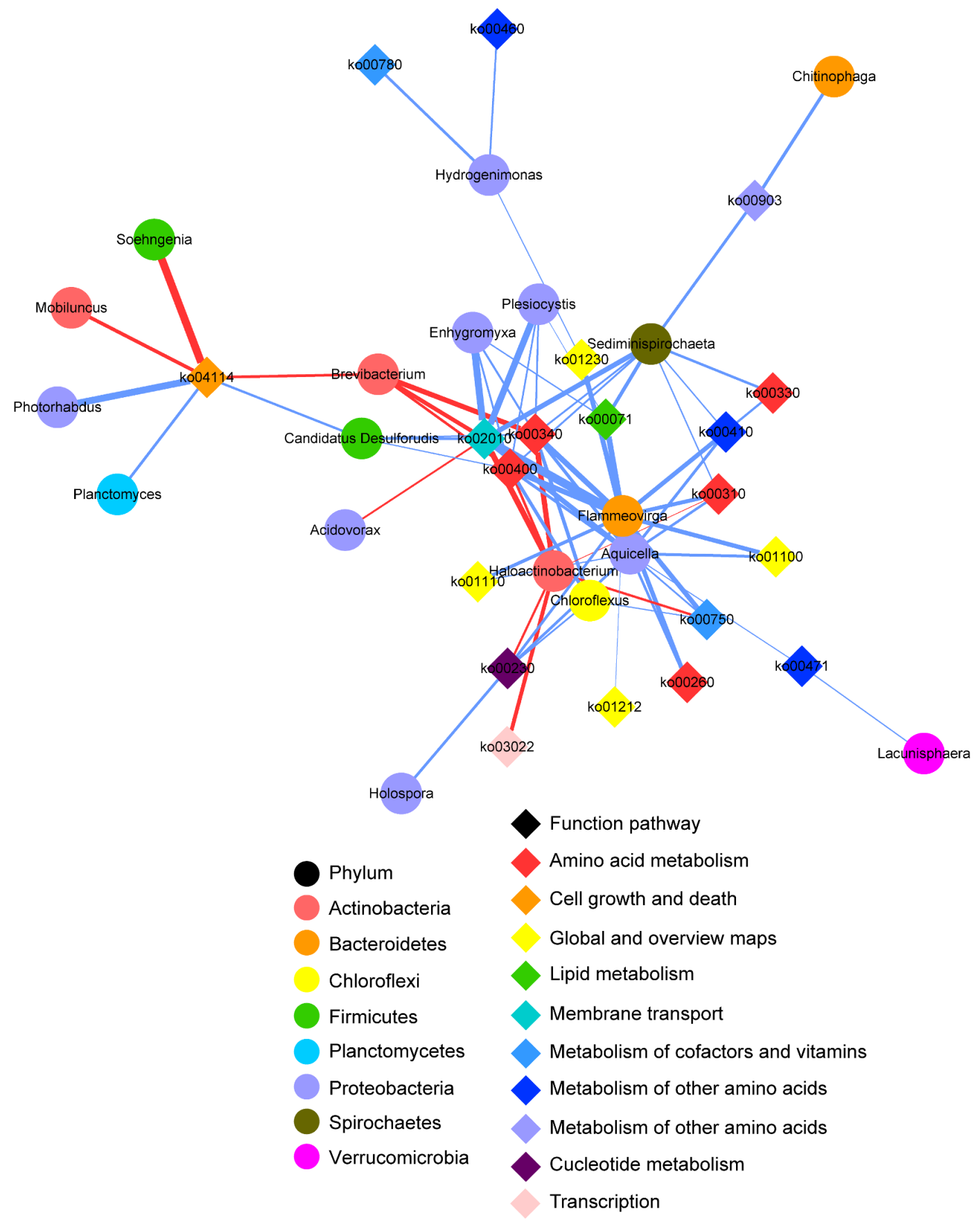

Fig. 5 Co-occurrence network shows the correlation between bacteria at genus level and KEGG functions based on the Spearman's rank correlation coefficients. The circles and diamonds represent microbial phyla and functions, respectively. Genera are categorized by phylum, and different colors represent different phyla and functions. The red lines indicate positive correlations (Spearman's $|r|>0.8, p<0.05$ ), and the blue lines indicate negative correlations (Spearman's $|r|>0.8, p<0.05$ ).

gradually recovered to a level similar to the control (Qian et al., 2007). Similar to the microbial community, RGs abundance in rhizosphere experienced significant change at $7 \mathrm{~d}$. In addition, many previous studies have shown that RGs abundance can easily change due to the input of various xenobiotics (Chen et al., 2016; Zhu et al., 2016; Wang et al., 2021).

In this study, many metabolism functions of rhizosphere soil microbiome significantly changed after continuous chlorine treatment. Besides, some functions were significantly upregulated by day 7 under $1 \mathrm{mg} \mathrm{L}^{-1}$ chlorine treatment, and recovered to no influence by day 14 , compared to the control group, such as caprolactam degradation (ko00930) and vitamin $B_{6}$ metabolism (ko00750).

Caprolactam degradation was related to xenobiotics biodegradation and metabolism and affected ecologically important soil bacteria (Bogatcheva et al., 2011); vitamin $B_{6}$ 


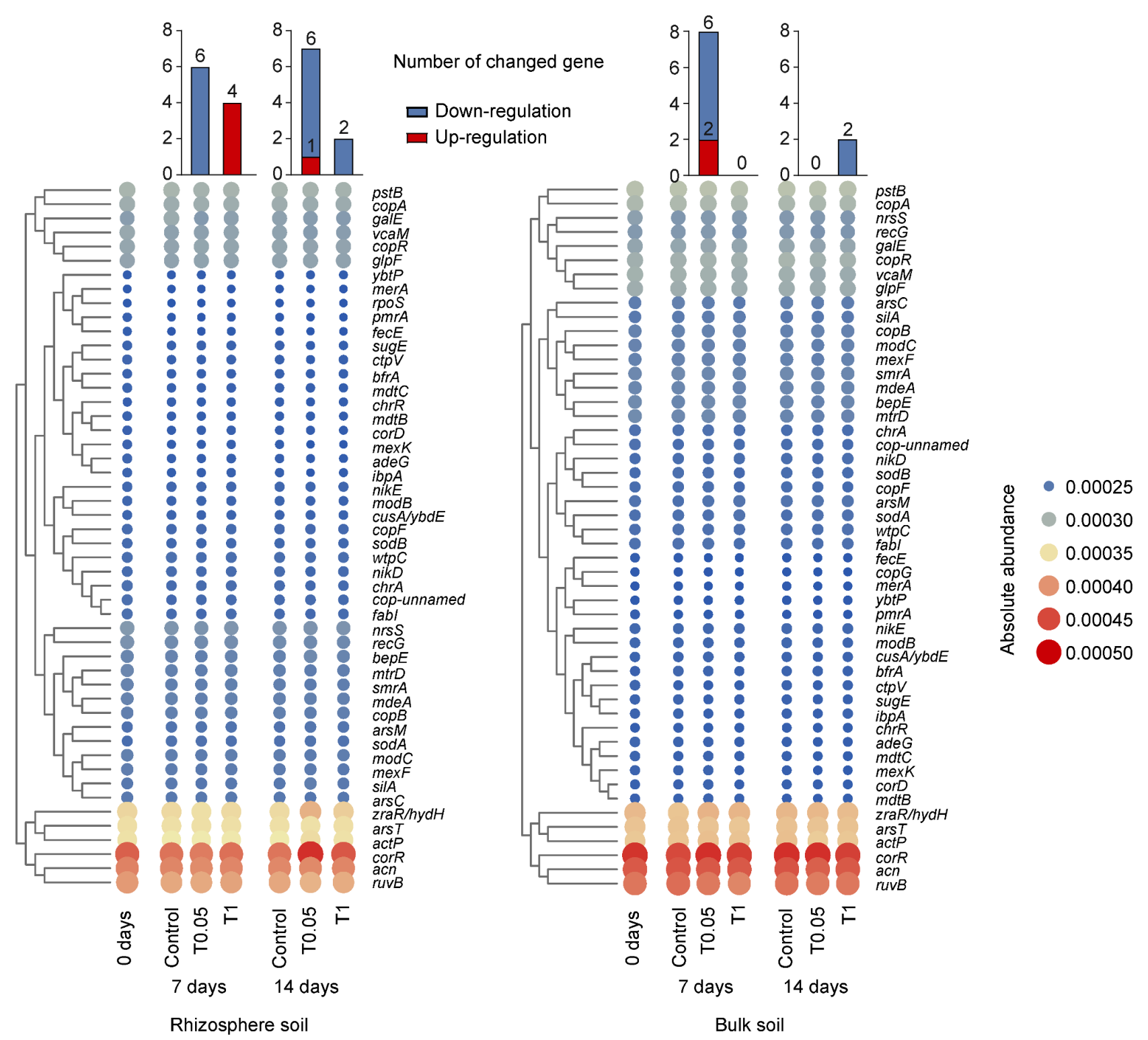

Fig. 6 Heatmap of top 50 abundant resistance genes (RGs) in rhizosphere or bulk soil after $14 \mathrm{~d}$ chlorine treatment. The bar graphs show the number of significantly changed in abundance genes under each treatment at $7 \mathrm{~d}$ and $14 \mathrm{~d}$ (the color of blue and red indicate the down and upregulated genes, respectively).

was an important coenzyme in life processes such as amino acid metabolism (ko09105), fatty acid metabolism (ko00071), and oxidative stress reaction (Rueschhoff, 2009). Consequently, rhizosphere microbes might be stimulated by chlorine treatment, and upregulated caprolactam degradation and vitamin $B_{6}$ metabolism pathways to protect themselves from damage until they adapted and recovered to the same level as control group. At the same time, several functions were significantly downregulated after $14-\mathrm{d} 0.05 \mathrm{mg} \mathrm{L}^{-1}$ chlorine treatment, indicating that these functions were damaged by chlorine. We assumed that the microbial community could drop into a "chlorine fertilizer trap," because chlorine benefits the growth of several plants (such as tobacco) to some extent (Podlena, 2009; Sun et al., 2017). Therefore, chlorine at low concentrations might be considered as a nutrient that was absorbed and transformed by the rhizosphere microbial community. Meanwhile, chlorine treatments damaged the microbial community structure with a delay, which means that continuous chlorine treatment promotes plants while it damages and perturbs the rhizosphere microbial community.

Co-occurrence network analysis was used to decipher the correlations between rhizosphere microbial genera and functions. The results showed that most of these correlations were negative, and a few were positive, of which Actinobacteria were significantly positively correlated with many metabolic functions. In this study, the abundance of neither of the above mentioned bacterial genera changed significantly in the rhizosphere soil after continuous chlorine treatment. Additionally, microbial function analysis indicated that the metabolic function of the microbial community was first 
upregulated by continuous chlorine treatment, and then recovered without any effect. Consequently, the co-occurrence network analysis of microbes and their functions supports the above conclusions about changes in the microbial community.

\section{Conclusions}

In summary, our study focused on the impact of continuous chlorine treatment on soil microbial community diversity, composition, and functions. Our results indicated that continuous chlorine treatment have no significant effect on the growth of wheat and soil microbiome neither in rhizosphere or in bulk soil over a period of time. Although enzyme activities, metabolism functions, and RGs abundance of rhizosphere microbiota might be affected slightly, the microbial community possesses capacity to adapt to the change of soil environment and recover to the level of control. In addition, the final chlorine concentrations were below detection limit. Therefore, it is considered that the continuous and wide application of sprayed chlorine-containing disinfectants only slightly threatens the soil ecosystem, mainly because of the quick degradation of chlorine in soil. However, the increasing usage of chlorine-containing disinfectants may further require research focused on its ecological risk in the long-term and in other ecosystems. For example, it is important to find out the limit of concentration of chlorine when applied to soil and when determining the suitable chlorine concentration interval of irrigation water. This work provided a comprehensive and systematic metagenomic method and ecological perspective for future studies on the ecological risk of chlorine-containing disinfectants or other pollutants.

\section{Acknowledgments}

This work was financially supported by the National Natural Science Foundation of China (41907210, 71903079, 21976161, 21777144).

\section{Electronic supplementary material}

Supplementary material is available in the online version of this article at https://doi.org/10.1007/s42832-022-0130-x and is accessible for authorized users.

\section{References}

Baxi, N.N., 2013. Influence of $\varepsilon$-caprolactam on growth and physiology of environmental bacteria. Annals of Microbiology 63, 1471-1476.

Bogatcheva, E., Dubuisson, T., Protopopova, M., Einck, L., Nacy, C. A., Reddy, V.M., 2011. Chemical modification of capuramycins to enhance antibacterial activity. Journal of Antimicrobial Chemother- apy $66,578-587$.

Bolger, A.M., Lohse, M., Usadel, B., 2014. Trimmomatic: a flexible trimmer for Illumina sequence data. Bioinformatics (Oxford, England) 30, 2114-2120.

Bulgarelli, D., Rott, M., Schlaeppi, K., van Themaat, E.V.L., Ahmadinejad, N., Assenza, F., Rauf, P., Huettel, B., Reinhardt, R., E., Schmelzer, J., Peplies, F.O., Gloeckner, R., Amann, T., Eickhorst, P., Schulze-Lefert, 2012. Revealing structure and assembly cues for Arabidopsis root-inhabiting bacterial microbiota. Nature 488, 91-95.

Chen, Q.L., An, X.L., Li, H., Su, J.Q., Ma, Y.B., Zhu, Y.G., 2016. Longterm field application of sewage sludge increases the abundance of antibiotic resistance genes in soil. Environment International 9293, 1-10.

China Huanqiu Web, 2020.02.20. Implementation of urban sewer disinfection, Wuhan has put in a total of 1963.58 tons of disinfectants, https://china.huanqiu.com/article/9CaKrnKptOt.

Choi, H.Y., Lee, Y.H., Lim, C.H., Kim, Y.S., Lee, I.S., Jo, J.M., Lee, H. Y., Cha, H.G., Woo, H.J., Seo, D.S., 2020. Assessment of respiratory and systemic toxicity of Benzalkonium chloride following a 14-day inhalation study in rats. Particle and Fibre Toxicology 17,5 .

Chu, W., Fang, C., Deng, Y., Xu, Z., 2021. Intensified disinfection amid COVID-19 pandemic poses potential risks to water quality and safety. Environmental Science \& Technology 55, 4084- 4086.

Cui, Y.X., Wang, X., Wang, X.X., Zhang, X.C., Fang, L.C., 2021. Evaluation methods of heavy metal pollution in soils based on enzyme activities: A review. Soil Ecology Letters 3, 169-177.

Dixon, P., 2003. VEGAN, a package of $R$ functions for community ecology. Journal of Vegetation Science 14, 927-930.

Duarte, R., Furtado, I., Sousa, L., Carvalho, C., 2020. The 2019 novel coronavirus (2019-nCoV): Novel virus, old challenges. Acta Medica Portuguesa 33, 33.

Feng, J.Y., Franks, A.E., Lu, Z.J., Xu, J.M., He, Y., 2021. Assembly and variation of root-associated microbiota of rice during their vegetative growth phase with and without lindane pollutant. Soil Ecology Letters 3, 207-219.

Fu, L.M., Niu, B.F., Zhu, Z.W., Wu, S.T., Li, W.Z., 2012. CD-HIT: accelerated for clustering the next-generation sequencing data. Bioinformatics (Oxford, England) 28, 3150-3152.

Hao, F.Z., Li, J.S., Wang, Z., Li, Y.F., 2018. Influence of chlorine injection on soil enzyme activities and maize growth under drip irrigation with secondary sewage effluent. Irrigation Science 36, 363-379.

Hu, B., Liang, D.L., Liu, J.J., Xie, J.Y., 2013. Ecotoxicological effects of copper and selenium combined pollution on soil enzyme activities in planted and unplanted soils. Environmental Toxicology and Chemistry 32, 1109-1116.

Huang, Y.L., Zeng, Y.H., Feng, H., Wu, Y.H., Xu, X.W., 2015. Croceicoccus naphthovorans sp. nov., a polycyclic aromatic hydrocarbons-degrading and acylhomoserine-lactone-producing bacterium isolated from marine biofilm, and emended description of the genus Croceicoccus. International Journal of Systematic and Evolutionary Microbiology 65, 1531-1536.

Huson, D.H., Auch, A.F., Qi, J., Schuster, S.C., 2007. MEGAN analysis of metagenomic data. Genome Research 17, 377-386. 
Krishnan, R., Menon, R.R., Likhitha, Busse, H.J., Tanaka, N., Krishnamurthi, S., Rameshkumar, N., 2017. Novosphingobium pokkalii sp. nov., a novel rhizosphere-associated bacterium with plant beneficial properties isolated from saline-tolerant pokkali rice. Research in Microbiology 168, 113-121.

Lee, Y., Jo, E., Lee, Y.J., Hettiaarachchi, S.A., Park, G.H., Lee, S.J., Heo, S.J., Kang, D.H., Oh, C., 2018. A novel glycosyl hydrolase family $16 \beta$-agarase from the agar-utilizing marine bacterium Gilvimarinus agarilyticus JEA5: the first agarase molecular and biochemical characterization in the genus Gilvimarinus. Journal of Microbiology and Biotechnology 28, 776-783.

Li, D.H., Liu, C.M., Luo, R.B., Sadakane, K., Lam, T.W., 2015. MEGAHIT: an ultra-fast single-node solution for large and complex metagenomics assembly via succinct de Bruijn graph. Bioinformatics (Oxford, England) 31, 1674-1676.

Li, P., Fan, X.Y., Qi, X.B., Fan, T., Zhao, Z.J., Zhao, X.F., 2013. Influence of soil residual nitrogen and coliforms in potato under alternate irrigation with chlorine reclaimed water. Chinese Agricultural Science Bulletin 29, 82-87.

Lonigro, A., Montemurro, N., Laera, G., 2017. Effects of residual disinfectant on soil and lettuce crop irrigated with chlorinated water. Science of the Total Environment 584, 595-602.

Lu, T., Ke, M.J., Lavoie, M., Jin, Y.J., Fan, X.J., Zhang, Z.Y., Fu, Z.W., Sun, L.W., Gillings, M., Penuelas, J., Qian, H., Zhu, Y.G., 2018. Rhizosphere microorganisms can influence the timing of plant flowering. Microbiome 6, 231.

Mendes, R., Garbeva, P., Raaijmakers, J.M., 2013. The rhizosphere microbiome: significance of plant beneficial, plant pathogenic, and human pathogenic microorganisms. FEMS Microbiology Reviews 37, 634-663.

Mincarelli, L., Vischetti, C., Craft, J., Tiano, L., 2016. DNA damage in different Eisenia andrei coelomocytes sub-populations after in vitro exposure to hydrogen peroxide. SpringerPlus 5, 1-7.

Miwa, N., Mitsuhashi, M., Kajiura, T., 2019. Screening of microorganisms producing a novel protein-asparaginase and characterization of the enzyme derived from Luteimicrobium album. Journal of Bioscience and Bioengineering 127, 281-287.

Murphy, R., Benndorf, R., de Beer, Z.W., Vollmers, J., Kaster, A.K., Beemelmanns, C., Poulsen, M., 2016. Comparative genomics reveals prophylactic and catabolic capabilities of actinobacteria within the fungus-farming termite symbiosis. MSphere 6, e01233 e20.

Olander, L.P., Vitousek, P.M., 2000. Regulation of soil phosphatase and chitinase activityby $\mathrm{N}$ and $\mathrm{P}$ availability. Biogeochemistry 49 , 175-191.

Oleńska, E., Maek, W., Wójcik, M., Swiecicka, I., Vangronsveld, J., 2020. Beneficial features of plant growth-promoting rhizobacteria for improving plant growth and health in challenging conditions: A methodical review. Science of the Total Environment 743, 140682.

Podlena, A., 2009. Effect of fertilization on content and uptake of chlorine by oilseed rape under pot experiment conditions. Journal of Elementology 14, 773-778.

Qian, H.F., Hu, B.L., Wang, Z.Y., Xu, X., Hong, T., 2007. Effects of validamycin on some enzymatic activities in soil. Environmental Monitoring and Assessment 125, 1-8.

Qian, H.F., Zhang, Q., Lu, T., Peijnenburg, W.J.G.M., Josep, P., Zhu, Y.
G., 2021. Lessons learned from COVID-19 on potentially pathogenic soil microorganisms. Soil Ecology Letters 3, 1-5.

Qu, Q., Zhang, Z.Y., Peijnenburg, W.J.G.M., Liu, W.Y., Lu, T., Hu, B.L., Chen, J., Chen, J., Lin, Z., Qian, H., 2020. Rhizosphere microbiome assembly and its impact on plant growth. Journal of Agricultural and Food Chemistry 68, 5024-5038.

Rangjaroen, C., Sungthong, R., Rerkasem, B., Teaumroong, N., Noisangiam, R., Lumyong, S., 2017. Untapped endophytic colonization and plant growth-promoting potential of the genus Novosphingobium to optimize rice cultivation. Microbes and Environments 32, 84-87.

Rueschhoff, E.E., 2009. Vitamin $B_{6}$ metabolism in Arabidopsis thaliana. Dissertations \& Theses-Gradworks, 3405145.

Shousei, K., Katsuhiko, F., 2018. Biochemical characteristics of cellulose and a green alga degradation by Gilvimarinus japonicas 12-2T, and its application potential for seaweed saccharification. Bioscience, Biotechnology, and Biochemistry 82, 1-7.

Subpiramaniyam, S., 2021. Outdoor disinfectant sprays for the prevention of COVID-19: Are they safe for the environment? Science of the Total Environment 759, 144289.

Sun, J.P., W, Z.H., Li, X.J., Ding, Y.F., Sun, H., Ping, W.L., 2017. Effect of high chlorine irrigation water on the chlorine absorption and distribution of potted flue-cured tobacco. Journal of Henan Agricultural Sciences 46, 44-48.

Truchado, P., Gil, M.I., Moreno-Candel, M., Allende, A., 2019. Impact of weather conditions, leaf age and irrigation water disinfection on the major epiphytic bacterial genera of baby spinach grown in an open field. Food Microbiology 78, 46-52.

Truchado, P., Gil, M.I., Suslow, T., Allende, A., Simon, V., 2018. Impact of chlorine dioxide disinfection of irrigation water on the epiphytic bacterial community of baby spinach and underlying soil. PLoS One 13, e0199291.

Wang, J., Zhang, B., Duan, H., Liang, C., Zhang, L., 2020. Key points of the program for disinfection technology in special places during the coronavirus disease-2019 (COVID-19) outbreak. China CDC Weekly 2, 140-142.

Wang, Y., Wang, X.J., Li, Y., Liu, Y.Y., Sun, Y., Xia, S.Q., Zhao, J.F., 2021. Effects of coexistence of tetracycline, copper and microplastics on the fate of antibiotic resistance genes in manured soil. Science of the Total Environment 790, 148087.

WHO, 2020a. WHO coronavirus disease (COVID-19) dashboard.

WHO, 2020b. Water, sanitation, hygiene, and waste management for SARS-CoV-2, the virus that causes COVID-19.

Yurkov, V., Stackebrandt, E., Holmes, A., Fuerst, J.A., Hugenholtz, P., Golecki, J., Gadon, N., Gorlenko, V.M., Kompantseva, E.I., Drews, G., 1994. Phylogenetic positions of novel aerobic, bacteriochlorophyll a-containing bacteria and description of Roseococcus thiosulfatophilus gen. nov., sp. nov., Erythromicrobium ramosum gen. nov., sp. nov., and Erythrobacter litoralis sp. nov. International Journal of Systematic and Evolutionary Microbiology 44, 427434.

Zhang, J., Zhang, C.W., Lei, Z., Jiang, J.Q., Kim, S.G., 2016. Novosphingobium oryzae sp. nov., a potential plant-promoting endophytic bacterium isolated from rice roots. International Journal of Systematic and Evolutionary Microbiology 66, 302-307.

Zhang, Z., Zhang, Q., Lu, T., Zhang, J., Sun, L., Hu, B., Hu, J., 
Peñuelas, J., Zhu, L., Qian, H., 2021. Residual chlorine disrupts the microbial communities and spreads antibiotic resistance in freshwater. Journal of Hazardous Materials 423, 127152.

Zhu, B., Chen, Q., Chen, S., Zhu, Y.G., 2016. Does organically produced lettuce harbor higher abundance of antibiotic resistance genes than conventionally produced? Environment International
98, 152-159.

Ziegler, S., Waidner, B., Itoh, T., Schumann, P., Spring, S., Gescher, J., 2013. Metallibacterium scheffleri gen. nov., sp. nov., an alkalinizing gammaproteobacterium isolated from an acidic biofilm. International Journal of Systematic and Evolutionary Microbiology 63, 1499-1504. 